\title{
Effect of Physio-biochemical Factors Influencing Moisture Stress Tolerance in Cotton (Gossypium hirsutum L.)
}

\author{
Thakur Pranita Prabhakar, D.P. Biradar* and I.S. Katageri
}

Department of Biotechnology, University of Agricultural Sciences, Dharwad - 580 005, India

*Corresponding author

\section{A B S T R A C T}

\begin{tabular}{|l|}
\hline Ke y w o r d s \\
$\begin{array}{l}\text { Cotton (Gossypium } \\
\text { hirsutum L.), } \\
\text { Moisture, Tolerance }\end{array}$ \\
\hline Article Info \\
\hline $\begin{array}{l}\text { Accepted: } \\
\text { 07 February } 2018 \\
\text { Available Online: } \\
\text { 10 March } 2018\end{array}$ \\
\hline
\end{tabular}

\section{Introduction}

Moisture stress incited by soil water deficit (Chaves et al., 2009) at reproductive stage of cotton (Michael et al., 1973; Quisenberry et al., 1985; Turner et al., 1986; Loka et al., 2012) is one of the reasons for reducing cotton productivity. India's cotton yield $568 \mathrm{~kg}$ per hectare continues to be lower than the global average of $800 \mathrm{~kg}$ per hectare (Anon., 2016). In India, maximum area of cotton cultivation, particularly hot and dry region of central and south zone under rainfed condition limits productivity, due to moisture stress. Irrigated cotton partially solves the problem in north India, where productivity is higher than rainfed condition (Anon., 2016). Therefore it
Physio-biochemical parameters were recorded under moisture stress and normal condition of tolerant and susceptible cotton $G$. hirsutum genotypes, when plants experiencing moisture stress at 65-67 DAS (15-17 days of water withholding and 80-82 DAS (30 days withholding). The genotypes, Khandwa-2, F-2226, RAJ-2, Bikaneri nerma, PH1009, CCH1831 and 5433A2A03N83 were found to exhibit one or more than one physiological parameters towards tolerance to higher RWC, less reduction in photosynthetic rate, tolerant conductance and transpiration rate with higher canopy temperature in drought proline and peroxidase enzyme activity play important role in exhibiting drought tolerance under moisture stress condition. This change in physio-biochemical process indirectly helps for increased yield potential in cotton genotypes, Khandwa-2, F-2226, 5433A2A03N83, RAJ-2, and RHC0811.

is not just sustainability but need of elevated production of cotton, it is the major challenge to meet the need of increasing world population under deteriorating arable land and depletion of water resources creating moisture stress. The identification of moisture stress tolerant cotton genotype based on physiobiochemical parameters with yield contributing traits to increase yield has been the major focus of researchers worldwide as a direct way of selection for breeding purpose (Rahman et al., 2008; Aktas et al., 2009; Brito et al., 2011; Ullah et al., 2017). Cotton genotypes tolerating moisture stress with low yielding ability were identified in several studies (Blum, 1988; Imran et al., 2012; Pettigrew, 2004; Kamaran et al., 2016). 
However, high yielding genotypes under water stress could likely to be low yielding under well-watered environments (Rosielle and Hamblin, 1981). Moreover the too dry on post-germination stage (Ananthi and Vijayaraghavan 2012; Karademir et al., 2009) probably do not reflect the conditions of natural drought. Even though drought avoidance (Kramer, 1983) of drought tolerance is almost impossible for increase productivity. Thus, identifying this traits for tolerance response, that can be assessed under both watered and water-stress conditions can characterize genotypes and may support cotton breeding programs in finding cultivars that are more tolerant to water stress or that may be used in the preliminary stages of a breeding program (Hassan et al., 2015; Kamaran et al., 2016; Zhang et al., 2010).

It has been known that plants started experiencing drought when soil water potential is less by more than 50 per cent in stressed plot than normal (Santos et al., 2011). Therefore in the present study observations were recorded only when the soil moisture of stressed plot was less by 50 per cent than normal. The physiological parameters included were canopy temperature, relative water content, transpiration rate, stomatal conductance and photosynthetic rate and chlorophyll content as an important attributes for moisture stress tolerance in cotton (Isoda et al., 2002; Massacci et al., 2008; Lahong et al., 2000). In the present study, RWC reflects the balance between water supply to the leaf tissue and transpiration rate through stomatal closure (Lugojan and Ciulca, 2011) and it results in reduction of transpiration rate which activated cooling system of leaf water potential and maintain higher canopy temperature leads to greater biomass production in dry land (Conaty et al., 2015). Lower transpiration rate along with higher relative water content (RWC) has been reported as selection criteria for plants against moisture stress (Malik et al., 1999; Rahman et $a l ., 2000)$. When plants expose to water stress, produce abscisic acid (ABA), which can promote stomatal closure by causing the efflux of solutes and water from the guard cells (Radin et al., 1988; Schroeder et al., 2001) and reduced stomatal conductance is positively associated for higher photosynthetic rate (Cornic and Massacci 1996; Tezara et al., 1999).

Moisture stress induces oxidative stress leads to increase production of reactive oxygen species (ROS), such as superoxide radicals (O2-), hydrogen peroxide $\left(\mathrm{H}_{2} \mathrm{O}_{2}\right)$ and hydroxyl radicals $(\mathrm{OH})$, which can attack lipid, proteins, carbohydrates and nucleic acid of plant system (Khatun et al., 2008). In order to eliminate ROS, plants increase activity of antioxidant enzymes peroxidase (Hosseini et al., 2015) that minimize cellular damage like oxidation of photosynthetic pigments and destruction of lipids, proteins and nucleic acids (Reddy et al., 2004). In addition to the production of antioxidants, osmotic adjustment occurs in plant cells through accumulation of compatible solutes like proline (Bray et al., 2000), regulate water loss by reducing the cell water potential (Fumis et al., 2002). Proline acts as an osmoregulator and cellular protectant under moisture stress (Hanower and Brzozowska, 1975) and it is variable in species according to factors genotypes (Patil et al., 2011). The increased osmoprotectant proline content and antioxidant peroxidase enzyme activity has been studied in different crops (Parida et al., 2007; Aktas et al., 2009; Amudha et al., 2014; Borgo et al., 2015; Marechaux et al., 2015; Jamal et al., 2015).

In cotton, the sensitivity to drought stress during flowering and boll development has been well established (Constable and Hearn, 1981; Cull et al., 1981; Turner et al., 1986; Loka et al., 2012) and insufficient soil water at 
this stage leads to a reduced plant height, number of fruiting branches, boll shedding, developed bolls and seed cotton yield (Pettigrew, 2004 and Ahuja et al., 2001; Karademir et al., 2011; Ananthi and Vijayaraghavan 2012; Loka et al., 2012). The amount of water utilized by cotton plants is related to the efficacy of physiological (Deeba et al., 2012) and biochemical processes (Hatfield et al., 1987) responsible for crop growth and yield. With all these viewpoints, the present investigation was planned to identify moisture stress tolerant cotton genotypes based on physio-biochemical and yield contributed traits towards moisture stress.

\section{Materials and Methods}

Plant materials, experimental site, location and design

Fourteen drought tolerant and one susceptible cotton genotype selected based on All India Co-ordinated Crop Improvement Project (AICCIP) report from the year of 1999-2014 were used. The seed of the 15 cotton $(G$. hirsutum L.) genotypes were collected from their respective breeding stations located in different ecological regions of India. List of the genotypes their source is given in Table 1 .

During kharif season of the year 2015-16, field experiment conducted at IABT Garden, UAS, Dharwad is situated in northern transitional zone of Karnataka altitude of 678 $\mathrm{m}$ above mean sea level with latitude $15^{0} 26^{1} \mathrm{~N}$ and longitude $76^{0} 7^{1}$ East. In the year of 201617 kharif, the same experimental taken in ARS, Dharwad Farm, Dharwad is situated in the northern transitional zone (Zone No. 8) of Karnataka with latitude of $15^{\circ} 46^{1}$ north and longitude of $75^{\circ} 0^{1}$ east altitude of $724 \mathrm{~m}$ above mean sea level (MSL), having similar agro climatic and rainfall as that of first location.
There were six blocks, in each block all 15 genotypes were sown randomly. Three blocks namely R4, R5 and R6 were used as control (maintain moisture at field capacity level) and another three blocks (R1, R2 and R3) later used to induce moisture stress. Each genotype was raised in a single row of $4.0 \mathrm{~m}$ length with a spacing $90 \times 20 \mathrm{~cm}$ in rain out shelter.

\section{Imposition of moisture stress}

Water was applied to entire field plot at field capacity up to 50 DAS (Days after sowing). To treated plot (considered to expose to moisture stress), after 50 DAS, watering was withheld to one plot (treated) which was separated by two layers of polythene sheets inserted up to $1-2 \mathrm{~m}$ in the soil to avoid lateral movement of water from one plot to another. The soil moisture content was measured in 10 random spots of soil depth $20 \mathrm{~cm}$ of entire plot using soil moisture meter at different days of plants leaf drooping response to moisture stress. One access tubes for Delta-T PR1 Profile probes were inserted into $1 \mathrm{~m}$ depth of soil bin. They were placed equidistant from the edge and $100 \mathrm{~cm}$ apart randomly. The mean of the 15 measurements was used to indicate the water content of the soil in the entire drought and control plot presented in Table 2. The plant response to moisture stress (50 per cent field capacity) was observed by various parameters at 65-67 DAS and 80-82 DAS. Rewatering was done to plot from 90 DAS onwards and continued to water till the end of the experiment and recorded yield contributed traits at harvesting.

\section{Physio-biochemical and productivity traits}

After induction of soil moisture stress (per cent reduction of soil moisture), treated plants were subjected to show leaf drooping and wilting symptoms due to decreasing leaf water deficiency (RWC) recorded by Barrs and Weatherly (1962) formula content $[(\mathrm{RWC}=$ 
[(FW-DW)/ (TW- DW) $] \times 100$ Where, FWfresh weight; DW- dry weight; TW- turgid weight (weight after the leaf was kept immersed in distilled water for $12 \mathrm{hr}$ )]. Other non-destructive physiological parameters such as chlorophyll content observed by SPAD meter (502 Plus, Spectrum Technologies, Plainfield, IL, USA) and stomatal conductance, transpiration rate and photosynthetic rate were recorded through IRGA (Infrared Red Gas Analyzers) system LI- 6400 (L1COR 6400, Lincoln Nebraska, USA).

The biochemical non-enzymatic and enzymatic process, like proline content and peroxidase enzyme activity were estimated by standard procedure (Bates et al., 1973 and Costa et al., (2002). During harvesting, productivity traits like number of sympodia, plant height, number of fruiting bodies/plant, number of harvested bolls/plant, per cent boll shedding and yield (kg/ha) were recorded. The data were analyzed statistically using standard protocols (Panse and Sukhatme 1967) and used Windows Stat 9.1 software for analyzing the data

\section{Results and Discussion}

\section{Induction of moisture stress at square formation stage}

In the world good crop of cotton can be raised with an annual rainfall of $800 \mathrm{~mm}$ distributed uniformly from March to November. There is sufficient moisture in soil to support normal germination, while delaying irrigation on this stage was found by several investigators as an effect on decreased yield (Singh et al., 1975; Grimes et al., 1970; Loka et al., 2012). This is occurred mainly due to soil water scarcity in central and southern region of India. In cotton the induction of reproductive parts (square) starts at 50-55 days after sowing depending on varieties within and between the species.
Flowering followed by squaring and finally boll setting continue to goes up to 120-180 days after sowing depending on species. Post germination moisture stress hinders the initial good growth, recovery may be expected but it depends on duration of moisture stress. In case of continued stage of moisture stress, crop may not recover at all then farmers will not continue to spend. In other situation, where crop experience moisture stress after normal establishment of crop leading to cause adverse effect on development of reproductive parts which might be enhance abscission of flowers and bolls and subsequently resulting in yield or quality loss (Ananthi and Vijayaraghavan 2012; Loka et al., 2012). In cotton, it is suggested that cotton plants experiencing moisture stress 15-30 days withholding water, considering this cotton plants experiencing moisture stress during 60-80 DAS is said to be one of the most important critical stages (Michael et al., 1973; Quisenberry et al., 1980).

It has also been known that plants started experiencing drought when soil water potential is less by more than 50 per cent in stressed plot than normal (Santos et al., 2011). In this study, after 15-17 days of water withholding (65-67 DAS), soil moisture content in stressed plots was 17.72 per cent reduced by 26.47 per cent over control condition and after 80-82 DAS (30-32 days water withholding), 8.9 per cent soil moisture content was recorded in stress induced plots. In comparison to control plot, 63.26 per cent reduction of moisture was recorded in stress induced plot (Table 2). Observations on different physio-biochemical traits were recorded when leaf relative water content at 65-67 DAS and 80-82 DAS in stressed plot was respectively less by 10.87 and 19.03 per cent than normal watered plots. Therefore these observations were helpful in identification of the plants experiencing moisture stress. 


\section{Physio-biochemical parameters}

After 15-17 days of water withholding (65-67 DAS), moisture stress effect in diverse cotton genotypes on physio-biochemical traits was studied. The significant difference was observed irrespective of genotypes between the conditions for several traits (Table 3). Although at 65-67 DAS, significant difference was not observed for transpiration rate and chlorophyll content (Table 3), after 30-32 days water withholding (80-82 DAS), ANOVA showed significant difference between conditions for all traits irrespective of genotypes (Table 4). To identify genetic variability under moisture stress, data was analyzed individually (condition wise separately), the significant difference was observed between the genotypes for physiobiochemical traits in moisture stress condition (Table 5).

Berger et al., (2010) reviewed that canopy temperature is one such integrative trait that reflects the plant water status or the resultant equilibrium between root water uptakes and shoot transpiration. Under stress condition canopy temperature is changing in cotton due to closure of stomata, reducing leaf activity, leaf area and increase leaf senescence (Marani et al., 1985) and it is affected by both the water status of plant (Meyer and walker, 1981) and the water status of soil (Wang et al., 2007). In this study, leaf senescence symptoms observed due to changes in plant and soil water status that leads to significant difference between the conditions for physiobiochemical traits. Under moisture stress condition (80-82 DAS), significant difference between genotypes observed for canopy temperature and the mean canopy temperature in moisture stress condition (29.17) was higher than control condition (27.94). Reddy et al., in 1996 reported that most advantageous canopy temperature ranged between 20 to $30^{\circ} \mathrm{C}$ in cotton. Lugojan and Ciulca (2011) reported the balance between water supply to the leaf tissue and transpiration rate is maintained through higher relative water content. During moisture stress, reduced transpiration rate activated cooling system of leaf water potential and maintain higher canopy temperature (Conaty et al., 2015). Although RWC was reduced after 30 days of water withholding, but their reduction rate was less in drought tolerant genotypes than susceptible MCU-5 in this study (Table 6). Therefore maintenance of higher RWC in drought tolerant genotypes (Sahana, RS-810, Khandwa-2, L-761, Bikaneri nerma and 5433A2A03N83) recorded higher canopy temperature than susceptible MCU-5. The susceptible MCU-5 recorded 31.42 per cent reduction of RWC at 80-82 DAS of moisture stress condition over control condition (Table 6). It indicates that higher RWC plays an important role for moisture stress resistance in drought tolerant genotypes. There are some studies reported, that the higher RWC in drought tolerant genotypes, had warmer canopy temperature than the sensitive genotypes in chickpea (Zaman-Allah et al., 2011), cowpea (Belko et al., 2012) and wheat (Rebetzke et al., 2013). Ananthi et al., (2012) observed lowest RWC (61.4) per cent in susceptible cotton genotype, "Surabhi" and highest in "KC2" (77.2), drought tolerant genotype. In this experiment also all drought tolerant cotton genotypes recorded higher RWC in stress condition (30 days of water withholding) than susceptible, MCU-5 (58.66).

Lower transpiration rate along with higher relative water content (RWC) has been reported as a selection criterion for plants against moisture stress (Malik et al., 1999; Rahman et al., 2000). Passioura (1982) and Zhang et al., (2010) implies a water conservation strategy by reducing transpiration rate, that preventing water loss from plant system leads to water saving for 
plant growth and helps to withstand in moisture stress condition. Isoda et al., (2002) reported, that reduction in transpiration rate under moisture stressed plants by 5-15 per cent than in well-watered plants, considered as typical for the field-grown cotton plants. Farquhar and Richards (1984) concluded, that under drought condition, low water use efficiency leads to decreased transpiration and at cellular level, abscisic acid was increased in shoots. Roberts and Dumbroff, (1986) reported, that the increase in levels of $\mathrm{ABA}$ was closely associated with a decrease in rate of transpiration. In this study decrease in transpiration rate was recorded in stress condition at 80-82 DAS than control condition (Table 6). But, per cent reduction rate of transpiration rate was less in drought tolerant genotypes PH1009 (2.74), F-2226 (2.32), Bikaneri nerma (4.03), JK-4 (4.53) and
Khandwa-2 (7.97) than susceptible MCU-5 (49.21). Previous studies in rose (Williams et al., 1999, 2000; Jenks et al., 2001) and tree tobacco (Cameron et al., 2006) reported, that plant adaptation in water deficit limit transpiration rate and delay the onset of cellular dehydration during prolonged drought (Kosma and Jenks, 2007).

When plants expose to water stress, produce abscisic acid (ABA), which can promote stomatal closure by causing the efflux of solutes and water from the guard cells (Radin et al., 1988; Schroeder et al., 2001). Gorham et al., (1998) reported that stomatal conductance was reduced by water deficit with consequent reductions in gas exchange parameters like net photosynthesis, transpiration and water use efficiency and an increase in leaf temperature of cotton.

Table.1 List of genotypes and their source of locations

\begin{tabular}{|l|l|l|}
\hline SI. No. & \multicolumn{1}{|c|}{ Genotype } & \multicolumn{1}{|c|}{ Source } \\
\hline P1 & Sahana & UAS, Dharwad, Karnataka \\
\hline P2 & RS-810 & RAU, Sriganganagar, Rajasthan \\
\hline P3 & Khandwa & Khandwa, Madhya-Pradesh \\
\hline P4 & L-761 & LAM, Guntur, Andhra-Pradesh \\
\hline P5 & GJHV-358 & Surat, Gujarat \\
\hline P6 & F-2226 & LAM, Guntur, Andhra-Pradesh \\
\hline P7 & JK-4 & JAU, Junagadh, Gujarat \\
\hline P8 & RAJ-2 & MPUAT, Udaipur, Rajasthan \\
\hline P9 & AK-23 & PDKV, Akola, Maharashtra \\
\hline P10 & Bikaneri nerma & PAU, Punjab \\
\hline P11 & PH 1009 & MAU, Nanded, Maharashtra \\
\hline P12 & CCH 1831 & NAU, Surat, Gujarat \\
\hline P13 & 5433A2A03N83 & CICR, Nagpur, Maharashtra \\
\hline P14 & MCU-5 (susceptible) & TNAU, Coimbatore, Tamilnadu \\
\hline P15 & RHC-0811 & MPKV, Rahuri, Maharashtra \\
\hline
\end{tabular}

Table.2 Percent of soil moisture content

\begin{tabular}{|l|c|c|c|}
\hline \multirow{2}{*}{ Conditions } & \multicolumn{3}{|c|}{ Days of interval } \\
\cline { 2 - 4 } & 50 DAS & 65 DAS & 80 DAS \\
\hline Control plots & 23.23 & 24.10 & 24.23 \\
\hline Moisture stress plots & 23.02 & 17.72 & 8.9 \\
\hline Percent reduction & 0.9 & 26.47 & 63.26 \\
\hline
\end{tabular}


Table.3 Analysis of variance for physio-biochemical traits in moisture stress and control condition at 65-67 DAS

\begin{tabular}{|c|c|c|c|c|c|c|c|c|c|}
\hline $\begin{array}{l}\text { Source of } \\
\text { variance }\end{array}$ & d.f. & $\mathrm{CT}\left({ }^{\circ} \mathrm{C}\right)$ & RWC (\%) & $\begin{array}{l}\text { TR (m mole of } \\
\mathrm{H}_{2} \mathrm{O} / \mathrm{m}^{2} / \mathrm{s} \text { ) }\end{array}$ & $\begin{array}{c}\mathrm{SC}(\boldsymbol{\mu} \text { mole } \\
\left.\mathrm{CO}_{2} / \mathrm{m}^{2} / \mathrm{s}\right)\end{array}$ & $\begin{array}{c}\text { PR }(\mu \text { mole } \\
\left.\mathrm{CO}_{2} / \mathrm{m}^{2} / \mathrm{s}\right)\end{array}$ & Chl (\%) & $\begin{array}{l}\text { Proline }(\mu g / g \\
\text { fresh wt.) }\end{array}$ & $\begin{array}{c}\text { GPOX (nM/min/g } \\
\text { protein) }\end{array}$ \\
\hline Replication & 1 & 1.11 & 30.88 & 0.31 & 0.004 & 0.84 & 31.45 & $2765.31 *$ & 301.17 \\
\hline $\begin{array}{c}\text { Genotypes } \\
\text { (G) }\end{array}$ & 14 & 0.56 & $34.35 * *$ & 0.39 & $0.009 * *$ & 2.58 & 5.6 & 311.93 & $7354.29 * *$ \\
\hline $\begin{array}{c}\text { Treatments } \\
\text { (T) }\end{array}$ & 1 & $32.07 * *$ & $1308.75^{* *}$ & 0.63 & $0.33 * *$ & $24.58 * *$ & 2.11 & $22502.06 * *$ & $630857.00 * *$ \\
\hline$T * G$ & 14 & 1.03 & 15.36 & 0.23 & 0.002 & 1.72 & 5.05 & 204.34 & $5831.92 * *$ \\
\hline Error & 30 & 0.7 & 8.57 & 0.33 & 0.003 & 2.47 & 5.53 & 157.13 & 1174.51 \\
\hline Total & 59 & 1.28 & 38.33 & 0.32 & 0.014 & 2.69 & 5.37 & 583.79 & 21872.24 \\
\hline
\end{tabular}

*, ** significant at $5 \%$ and $1 \%$ respectively

Table.4 Analysis of variance for physio-biochemical traits in moisture stress and control condition at 80-82 DAS (Analyzed condition wise)

\begin{tabular}{|c|c|c|c|c|c|c|c|c|c|}
\hline $\begin{array}{c}\text { Source of } \\
\text { variance }\end{array}$ & d.f. & $\mathrm{CT}\left({ }^{\circ} \mathrm{C}\right)$ & RWC (\%) & $\begin{array}{c}\text { TR (m mole of } \\
\left.\mathrm{H}_{2} \mathrm{O} / \mathrm{m}^{2} / \mathrm{s}\right)\end{array}$ & $\begin{array}{c}\mathrm{SC}(\mu \mathrm{mole} \\
\left.\mathrm{CO}_{2} / \mathrm{m}^{2} / \mathrm{s}\right)\end{array}$ & $\begin{array}{c}\mathrm{PR}(\boldsymbol{\mu} \text { mole } \\
\left.\mathrm{CO}_{2} / \mathrm{m}^{2} / \mathrm{s}\right)\end{array}$ & Chl (\%) & $\begin{array}{l}\text { Proline ( } \mu g / g \\
\text { fresh wt.) }\end{array}$ & $\begin{array}{c}\text { GPOX (nM/min/g } \\
\text { protein) }\end{array}$ \\
\hline Replication & 1 & 96.35 & 26.45 & 3.32 & 0.025 & 4.25 & 1.1 & 8.06 & 74309.12 \\
\hline $\begin{array}{c}\text { Genotypes } \\
(\mathrm{G}) \\
\end{array}$ & 14 & $1.45^{*}$ & $19.18^{*}$ & $2.21 *$ & $0.006^{*}$ & $7.06^{*}$ & $6.435^{*}$ & $199.78 * *$ & $35327.36^{*}$ \\
\hline Conditions & 1 & $22.51 * *$ & $4063.58 * *$ & $26.41 * *$ & $0.23 * *$ & $141.87 * *$ & $150.21 * *$ & $35869.30 * *$ & $2144364.37 * *$ \\
\hline$T * G$ & 14 & 1.77 & 14.01 & 1.35 & $0.028 * *$ & $10.89 * *$ & 4.31 & $192.02 * *$ & $47353.48 * *$ \\
\hline Error & 30 & 4.34 & 7.78 & 1.04 & 0.011 & 3.08 & 1.74 & 51.04 & 16637.25 \\
\hline Total & 59 & 3.35 & 80.71 & 1.82 & 0.02 & 8.23 & 5.98 & 726.88 & 64423.96 \\
\hline
\end{tabular}

*, ** significant at $5 \%$ and $1 \%$ respectively

CT- Canopy temperature $\left({ }^{\circ} \mathrm{C}\right)$; RWC- Relative water content (percent gm of leaf sample); Chl- Chlorophyll content (\% leaf area); SC-Stomatal conductance ( $\mu$ mole $\left.\mathrm{CO}_{2} / \mathrm{m}^{2} / \mathrm{s}\right)$; TR- Transpiration rate (m mole of $\left.\mathrm{H}_{2} \mathrm{O} / \mathrm{m}^{2} / \mathrm{s}\right) ;$ PR- Photosynthetic rate $\left(\mu\right.$ mole $\left.\mathrm{CO}_{2} / \mathrm{m}^{2} / \mathrm{s}\right) ;$ Proline $(\mu \mathrm{g} / \mathrm{g}$ fresh wt.); GPOX- Peroxidase activity $(\mathrm{nM} / \mathrm{min} / \mathrm{g}$ protein) 
Table.5 Analysis of variance for physio-biochemical traits in moisture stress condition at 80-82 DAS (Analyzed condition wise)

\begin{tabular}{|c|c|c|c|c|c|c|c|c|c|}
\hline $\begin{array}{l}\text { Source of } \\
\text { variance }\end{array}$ & d.f. & $\mathrm{CT}\left({ }^{\circ} \mathrm{C}\right)$ & RWC (\%) & $\begin{array}{c}\text { TR (m mole of } \\
\left.\mathrm{H}_{2} \mathrm{O} / \mathrm{m}^{2} / \mathrm{s}\right)\end{array}$ & $\begin{array}{l}\mathrm{SC}(\mu \text { mole } \\
\left.\mathrm{CO}_{2} / \mathrm{m}^{2} / \mathrm{s}\right)\end{array}$ & $\begin{array}{l}\text { PR }(\mu \text { mole } \\
\left.\mathrm{CO}_{2} / \mathrm{m}^{2} / \mathrm{s}\right)\end{array}$ & Chl (\%) & $\begin{array}{l}\text { Proline }(\mu \mathrm{g} / \mathrm{g} \\
\text { fresh wt.) }\end{array}$ & $\begin{array}{c}\text { GPOX (nM/min/g } \\
\text { protein) }\end{array}$ \\
\hline Replication MSS & 1 & 49 & 7.41 & 0.06 & 0.034 & 0.02 & 0.4 & 303.15 & 51706.01 \\
\hline Genotype MSS & 14 & $2.70^{*}$ & $28.66^{* * *}$ & $2.02 *$ & $0.019^{* *}$ & $15.66^{* *}$ & $9.04 * *$ & $366.22 * *$ & 71078.33** \\
\hline Error MSS & 14 & 1.49 & 11.09 & 1 & 0.008 & 4.28 & 2.16 & 34.57 & 23122.67 \\
\hline C. V. & & 4.18 & 4.76 & 19.39 & 19.75 & 11.52 & 3.56 & 5.17 & 13.54 \\
\hline C. D. $5 \%$ & & 2.61 & 7.14 & 2.14 & 0.2 & 4.44 & 3.15 & 12.61 & 326.14 \\
\hline S.Em. \pm & & 0.86 & 2.36 & 0.71 & 0.07 & 1.46 & 1.04 & 4.16 & 107.52 \\
\hline
\end{tabular}

*, ** significant at $5 \%$ and $1 \%$ respectively

CT- Canopy temperature $\left({ }^{\circ} \mathrm{C}\right)$; RWC- Relative water content (percent gm of leaf sample); Chl- Chlorophyll content (\% leaf area); SC- Stomatal conductance $(\mu$ mole $\left.\mathrm{CO}_{2} / \mathrm{m}^{2} / \mathrm{s}\right)$; TR- Transpiration rate (m mole of $\left.\mathrm{H}_{2} \mathrm{O} / \mathrm{m}^{2} / \mathrm{s}\right)$; PR- Photosynthetic rate $\left(\mu\right.$ mole $\left.\mathrm{CO}_{2} / \mathrm{m}^{2} / \mathrm{s}\right)$; Proline $(\mu \mathrm{g} / \mathrm{g}$ fresh wt.); GPOX- Peroxidase activity $(\mathrm{nM} / \mathrm{min} / \mathrm{g}$ protein)

Table.6 Physiological traits in cotton genotypes after 30-32 days of water withholding (80-82 DAS)

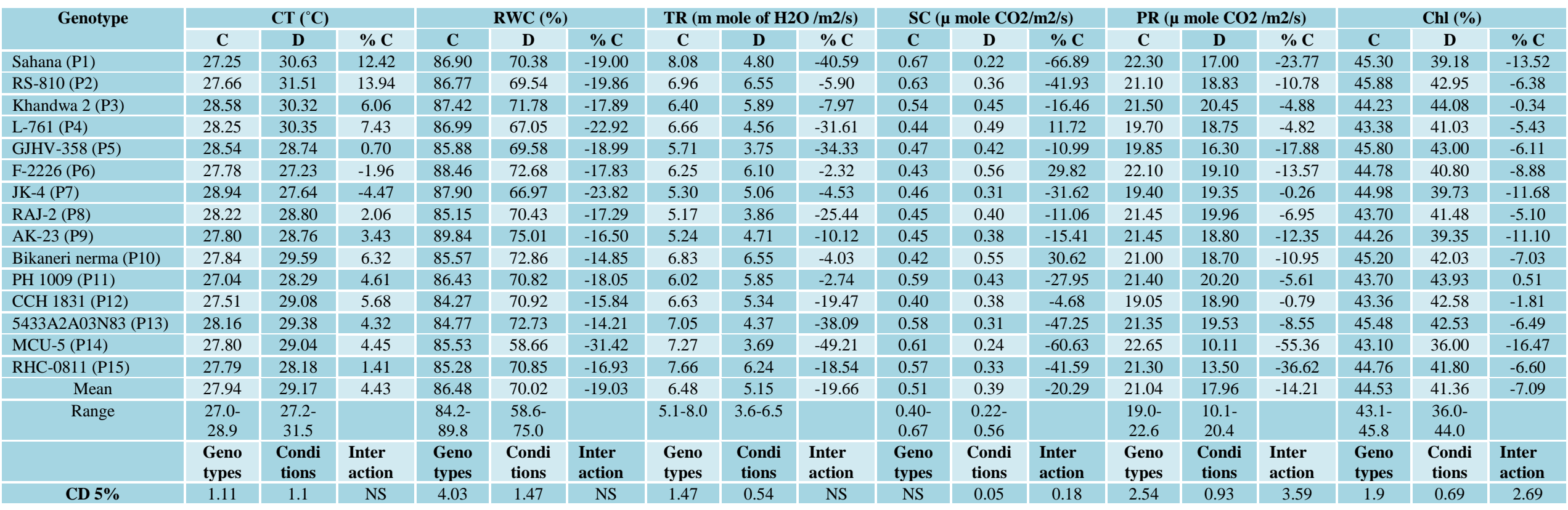

C-Normal condition, D-moisture stress condition, \% C- per cent change 
Table.7 Biochemical traits in cotton genotypes after 30-32 days of water withholding (80-82 DAS)

\begin{tabular}{|l|c|c|c|c|c|c|c|}
\hline \multirow{2}{*}{ Genotype } & \multicolumn{3}{|c|}{ Proline content $(\boldsymbol{\mu g}$ /g fresh wt) } & \multicolumn{3}{c|}{ GPOX (nM/min/g protein) } \\
\hline & C & D & \% C & C & D & \% C \\
\hline Sahana (P1) & 61.7 & 102 & 65.22 & 834.6 & 1000.5 & 19.88 \\
\hline RS-810 (P2) & 68.1 & 94 & 38.03 & 880.2 & 927.7 & 5.4 \\
\hline Khandwa 2 (P3) & 62.4 & 119.7 & 91.72 & 774.8 & 1201.1 & 55.02 \\
\hline L-761 (P4) & 57.9 & 106.2 & 83.6 & 640.3 & 1046.1 & 63.37 \\
\hline GJHV-358 (P5) & 65.1 & 142.7 & 119.25 & 647.7 & 1150.3 & 77.6 \\
\hline F-2226 (P6) & 65.9 & 121.7 & 84.7 & 719.9 & 1184 & 64.46 \\
\hline JK-4 (P7) & 64.5 & 107.3 & 66.36 & 749.5 & 887.1 & 18.36 \\
\hline RAJ-2 (P8) & 60.1 & 116.2 & 93.37 & 664.4 & 1448.6 & 118.03 \\
\hline AK-23 (P9) & 62.1 & 119.1 & 91.83 & 809.5 & 1275.5 & 57.57 \\
\hline Bikaneri nerma & 66.8 & 110.2 & 64.88 & 634.6 & 1215.7 & 91.58 \\
\hline (P10) & & & & & & \\
\hline PH 1009 (P11) & 69.4 & 122.3 & 76.37 & 821.8 & 1247.7 & 51.82 \\
\hline CCH 1831 & 66.5 & 131 & 97.02 & 798.9 & 1041.6 & 30.39 \\
\hline (P12) & & & & & & \\
\hline 5433A2A03N83 & 71.5 & 109.2 & 52.6 & 736.7 & 1366 & 85.42 \\
\hline (P13) & & & & & & \\
\hline MCU-5 (P14) & 65.1 & 90.2 & 38.55 & 710.2 & 731.6 & 3.01 \\
\hline RHC-0811 & 64 & 112.7 & 76.15 & 747.8 & 1118.8 & 49.61 \\
\hline (P15) & & & & & & \\
\hline \multicolumn{1}{|c|}{ Mean } & 64.74 & 113.65 & 75.98 & 744.72 & 1122.82 & 52.77 \\
\hline \multicolumn{1}{|c|}{ Range } & $57.8-71.5$ & $90.1-$ & & $634.5-$ & $731.5-$ & \\
\hline CD 5\% & 13.89 & 5.07 & 19.65 & 250.82 & 91.59 & 354.71 \\
\hline & & 142.7 & & 880.2 & 1448.5 & \\
\hline & & & & & & \\
\hline
\end{tabular}


Table.8 Analysis of variance for productivity traits in moisture stress and control condition at harvesting stage

\begin{tabular}{|c|c|c|c|c|c|c|c|}
\hline $\begin{array}{l}\text { Source of } \\
\text { variance }\end{array}$ & d.f. & $\begin{array}{c}\text { No. of } \\
\text { sympodia/ } \\
\text { plant }\end{array}$ & Plant height & $\begin{array}{l}\text { No. of fruit } \\
\text { bodies/plant }\end{array}$ & No. of bolls/plant & $\begin{array}{c}\text { Boll shedding } \\
(\%)\end{array}$ & Yield (kg/ha) \\
\hline Replication & 1 & $81.95^{*}$ & 22.2 & 12.8 & $3.70 *$ & 0.017 & $561129.44 *$ \\
\hline $\begin{array}{c}\text { Genotypes } \\
(\mathbf{G})\end{array}$ & 14 & 7.87 & $165.74 *$ & $12.38 * *$ & $4.61 *$ & $39.85^{*}$ & $178430.98 * *$ \\
\hline $\begin{array}{c}\text { Treatments } \\
\text { (T) }\end{array}$ & 1 & $765.94 * *$ & 3788.18 & $1126.41 * *$ & $144.43 * *$ & $2702.31 * *$ & $2791162.63 * *$ \\
\hline$T * G$ & 14 & 4.92 & 63.24 & $15.26 * *$ & 5.67 & $61.57 * *$ & 47513.07 \\
\hline Error & 30 & 8.79 & 78.1 & 4.21 & 1.51 & 10.83 & 25911.59 \\
\hline Total & 59 & 20.49 & 158.25 & 27.8 & 5.66 & 75.37 & 114097.1 \\
\hline
\end{tabular}

$*, * *$ significant at $5 \%$ and $1 \%$ respectively

Table.9 Analysis of variance for productivity traits in moisture stress condition at harvesting stage (Analyzed condition wise)

\begin{tabular}{|c|c|c|c|c|c|c|c|}
\hline $\begin{array}{l}\text { Source of } \\
\text { variance }\end{array}$ & d.f. & $\begin{array}{c}\text { No. of } \\
\text { sympodia/ } \\
\text { plant }\end{array}$ & Plant height & $\begin{array}{l}\text { No. of fruit } \\
\text { bodies/plant }\end{array}$ & No. of bolls/plant & $\begin{array}{c}\text { Boll shedding } \\
(\%)\end{array}$ & Yield (kg/ha) \\
\hline $\begin{array}{l}\text { Replication } \\
\text { MSS }\end{array}$ & 1 & 0.41 & 14.18 & 7.5 & 0.97 & 67.71 & 153184.4 \\
\hline $\begin{array}{l}\text { Genotype } \\
\text { MSS } \\
\end{array}$ & 14 & 3.22 & $93.24 *$ & $18.31 * *$ & $5.46 * *$ & $91.82 * *$ & $75849.24 * *$ \\
\hline Error MSS & 14 & 1.92 & 85.31 & 2.21 & 0.41 & 11.6 & 1012.25 \\
\hline C. V. & & 8.82 & 8 & 10.33 & 6.12 & 7.75 & 3.19 \\
\hline C.D. $5 \%$ & & 2.97 & 19.81 & 3.19 & 1.37 & 7.3 & 68.24 \\
\hline S.Em. \pm & & 0.98 & 6.53 & 1.05 & 0.45 & 2.41 & 22.5 \\
\hline
\end{tabular}


Table.10 Yield and yield contributing traits in cotton genotypes at harvesting stage

\begin{tabular}{|c|c|c|c|c|c|c|c|c|c|c|c|c|c|c|c|c|c|c|}
\hline \multirow[t]{2}{*}{ Genotype } & \multicolumn{3}{|c|}{ No. of sympoida/ plant } & \multicolumn{3}{|c|}{ Plant height (cm) } & \multicolumn{3}{|c|}{ No. of fruiting bodies/plant } & \multicolumn{3}{|c|}{ No. of bolls harvested/plant } & \multicolumn{3}{|c|}{$\%$ boll shedding } & \multicolumn{3}{|c|}{ Yield (kg/ha) } \\
\hline & $\mathbf{C}$ & D & $\% \mathrm{C}$ & $\mathrm{C}$ & D & $\% \mathrm{C}$ & $\mathbf{C}$ & D & $\% \mathrm{C}$ & $\mathrm{C}$ & D & $\% \mathrm{C}$ & $\mathbf{C}$ & D & $\% \mathrm{C}$ & $\mathbf{C}$ & D & $\% \mathrm{C}$ \\
\hline Sahana (P1) & 26.69 & 16.44 & -38.41 & 136.19 & 119.19 & -12.48 & 24.28 & 15.63 & -35.63 & 13.90 & 10.90 & -21.58 & 31.1 & 43.4 & 39.53 & 1955.7 & 1034 & -47.13 \\
\hline RS-810 (P2) & 19.5 & 16.13 & -17.31 & 130.44 & 111.25 & -14.71 & 22.98 & 19.7 & -14.25 & 12.20 & 13.80 & 13.11 & 27.7 & 43.4 & 56.65 & 1031.3 & 883.6 & -14.32 \\
\hline Khandwa 2 (P3) & 19.44 & 15.06 & -22.51 & 135.81 & 112.31 & -17.3 & 20.25 & 20.15 & -0.49 & 12.30 & 13.80 & 12.2 & 29.2 & 44.1 & 51.12 & 1482.7 & 1213.9 & -18.13 \\
\hline L-761 (P4) & 21.38 & 15.44 & -27.78 & 138.81 & 124.31 & -10.45 & 20.27 & 13.59 & -32.98 & 11.65 & 9.60 & -17.56 & 28.6 & 50.3 & 76.01 & 1309.6 & 1135.3 & -13.31 \\
\hline GJHV-358 (P5) & 22.31 & 17.06 & -23.53 & 143.25 & 125.25 & -12.57 & 21.25 & 14.1 & -33.65 & 12.80 & 9.00 & -29.69 & 29 & 42.6 & 46.95 & 1126.1 & 967.6 & -14.08 \\
\hline F-2226 (P6) & 20.25 & 16.13 & -20.37 & 113.44 & 103.25 & -8.98 & 21.1 & 12.08 & -42.77 & 12.20 & 9.80 & -19.67 & 30.4 & 51.3 & 68.96 & 1361.2 & 959.4 & -29.52 \\
\hline JK-4 (P7) & 21.88 & 14.38 & -34.29 & 132.56 & 118.38 & -10.7 & 24.29 & 10.98 & -54.81 & 13.90 & 9.20 & -33.81 & 27.6 & 50.1 & 81.57 & 1403.3 & 784.5 & -44.09 \\
\hline RAJ-2 (P8) & 23.75 & 15.88 & -33.16 & 132 & 125.69 & -4.78 & 23.83 & 12.44 & -47.8 & 12.80 & 9.50 & -25.78 & 27.7 & 49.3 & 77.98 & 1969.8 & 1410.5 & -28.39 \\
\hline AK-23 (P9) & 24.81 & 16.25 & -34.51 & 139.13 & 112.88 & -18.87 & 24.25 & 12.49 & -48.51 & 13.80 & 9.30 & -32.61 & 31.5 & 36.2 & 14.77 & 1286.1 & 878.1 & -31.73 \\
\hline Bikaneri nerma $(\mathrm{P} 10)$ & 22.5 & 14.75 & -34.44 & 120.44 & 110 & -8.67 & 20.33 & 13.61 & -33.03 & 12.10 & 9.60 & -20.66 & 32.2 & 38.1 & 18.49 & 1289.4 & 921.3 & -28.55 \\
\hline PH 1009 (P11) & 21.81 & 13.19 & -39.54 & 130.63 & 109.25 & -16.36 & 25.19 & 13.95 & -44.62 & 16.10 & 9.00 & -44.1 & 33.5 & 31.2 & -7.06 & 1455.4 & 1002.4 & -31.13 \\
\hline CCH 1831 (P12) & 24.44 & 15.19 & -37.85 & 140.88 & 108.94 & -22.67 & 21.35 & 13.86 & -35.07 & 12.40 & 9.80 & -20.97 & 32.7 & 35.2 & 7.61 & 1400.1 & 1119.9 & -20.01 \\
\hline 5433A2A03N83 (P13) & 23.75 & 17.38 & -26.84 & 125.25 & 118.81 & -5.14 & 25.04 & 18.84 & -24.76 & 14.80 & 12.30 & -16.89 & 31.7 & 41.6 & 31.1 & 1423.5 & 1009.9 & -29.06 \\
\hline MCU-5 (P14) & 25.06 & 14.31 & -42.89 & 127.19 & 111.06 & -12.68 & 24.43 & 10.01 & -59.01 & 15.00 & 9.30 & -38 & 34.4 & 55.2 & 60.17 & 1169.6 & 557.7 & -52.32 \\
\hline RHC-0811 (P15) & 25.13 & 17.94 & -28.61 & 123.56 & 120.63 & -2.38 & 27.18 & 14.59 & -46.32 & 16.70 & 11.20 & -32.93 & 30.9 & 47.6 & 54.25 & 1744.9 & 1060.2 & -39.24 \\
\hline Mean & 22.85 & 15.7 & -30.8 & 131.3 & 115.41 & -11.92 & 23.07 & 14.4 & -36.91 & 13.51 & 10.41 & -21.93 & 30.54 & 43.96 & 45.21 & 1427.25 & 995.89 & -29.4 \\
\hline \multirow[t]{2}{*}{ Range } & $19.4-26.6$ & $\begin{array}{l}13.1- \\
17.9\end{array}$ & & $\begin{array}{l}113.4- \\
143.2\end{array}$ & $\begin{array}{c}103.2- \\
125.6\end{array}$ & & $20.2-27.1$ & $\begin{array}{l}10.0- \\
20.1\end{array}$ & & $11.6-16.7$ & $\begin{array}{l}9.0- \\
13.8\end{array}$ & & $27.6-34.4$ & $\begin{array}{c}31.2- \\
55.2\end{array}$ & & $\begin{array}{c}1031.3- \\
1969.7\end{array}$ & $\begin{array}{l}557.7- \\
1410.5\end{array}$ & \\
\hline & $\begin{array}{l}\text { Geno } \\
\text { types }\end{array}$ & $\begin{array}{c}\text { Condi } \\
\text { tions }\end{array}$ & $\begin{array}{l}\text { Inter } \\
\text { action }\end{array}$ & $\begin{array}{l}\text { Geno } \\
\text { types }\end{array}$ & $\begin{array}{c}\text { Condi } \\
\text { tions }\end{array}$ & $\begin{array}{l}\text { Inter } \\
\text { action }\end{array}$ & Geno types & $\begin{array}{c}\text { Condi } \\
\text { tions }\end{array}$ & $\begin{array}{l}\text { Inter } \\
\text { action }\end{array}$ & Geno types & $\begin{array}{c}\text { Condi } \\
\text { tions }\end{array}$ & $\begin{array}{l}\text { Inter } \\
\text { action }\end{array}$ & $\begin{array}{l}\text { Geno } \\
\text { types }\end{array}$ & $\begin{array}{c}\text { Condi } \\
\text { tions }\end{array}$ & $\begin{array}{l}\text { Inter } \\
\text { action }\end{array}$ & $\begin{array}{l}\text { Geno } \\
\text { types }\end{array}$ & $\begin{array}{c}\text { Condi } \\
\text { tions }\end{array}$ & $\begin{array}{l}\text { Inter } \\
\text { action }\end{array}$ \\
\hline CD 5\% & NS & 1.56 & NS & 12.76 & 4.66 & NS & 2.96 & 1.08 & 4.19 & 1.78 & 0.65 & 2.51 & 4.75 & 1.74 & 6.72 & 232.46 & 84.88 & NS \\
\hline
\end{tabular}

C-Normal condition, D-moisture stress condition, \% C-per cent change 
Massacci et al., (2008) found irrigated plants had greater stomatal conductance than dryland cotton and 30 per cent reduction of stomatal conductance observed in stressed plants as compared to non-stressed (Khan et al., 2015). It shows the blocking of the stomata to reduce water loss and increase adaptability of plants to stress condition. Therefore in this study, all genotypes showed decreased stomatal conductance at 80-82 DAS of moisture stress condition than control condition (Table 6).

However in moisture stress condition, per cent reduction of stomatal conductance was less in drought tolerant genotypes RAJ-2 (11.06), AK-23 (15.41), CCH-1831 (4.68), GJHV-358 (10.99), Khandwa-2 (16.46) and RAJ-2 (31.62) than susceptible MCU-5 (60.63). Contrastingly, in some genotypes, L761 (11.72), F-2226 (29.82) and Bikaneri nerma (30.62) higher per cent of stomatal conductance recorded in water deficit condition than watered condition, probably as a result of a higher capacity for water uptake in this genotypes. In cotton many studies reported, as soil water availability diminished, stomatal conductance decrease (Lopez et al., 1995; Laffray and Louguet, 1990; Costa and Cothren 2011; Cornish et al., 2016).

Photosynthesis is closely related to water and carbon (C) metabolism in plants, moisture stress is known to reduce photosynthetic rate by decreased RWC and is mainly through stomatal closure or metabolic impairment (Cornic and Massicci, 1996). Many studies observed significant inhibition of photosynthetic rate in cotton under a waterlimited environment (Turner et al., 1986; Leidi et al., 1993). In this study, all cotton genotypes recorded lower photosynthetic rate at 30 days of water withholding (80-82 DAS) than control condition (Table 6). Under severe water stress when relative water contents drops below 70 per cent, inhibition of photosynthesis is attributed to non-stomata1 effects (Kaiser, 1987; Ennahli and Earl, 2005), therefore higher reduction of photosynthetic rate observed, might be due to reduction of chlorophyll content (Table 6). Nepomuceno et al., (1998) demonstrated that the tolerant genotypes were able to maintain higher photosynthesis, stomatal conductance and relative water content near unstressed control levels. Similarly in this study drought tolerant genotypes CCH1831 (0.79), JK-4 (0.26), L-761 (4.82), Khandwa-2 (4.88), PH1009 (5.61) and 5433A2A03N83 (8.55) recorded smaller reduction of photosynthetic rate than susceptible MCU-5 (55.36) in moisture stress condition. Reduction in stomatal conductance in susceptible MCU-5 is to save water for survival, but due to reduced photosynthesis, susceptible genotype MCU-5 recorded very low yield. Therefore less reduction of photosynthetic rate in drought tolerant genotype under moisture stress condition (80-82 DAS) is considered a decisive factor for higher cotton production (Lopez et al., 1995; Ullah et al., 2008).

Plant chlorophyll content is generally controlled and there was wide genetic variability existed among all crop species. However, irrespective of genotypes, moisture stress reduces the chlorophyll content in crop plants. The extent of chlorophyll content is again depend on genotypes and their level of moisture stress tolerance (Yi et al., 2000; Li et al., 2012; Li et al., 2014).

In the present study after 30 days of water withholding (80-82 DAS), all cotton genotypes showed reduced chlorophyll content than control condition and in drought tolerant genotypes Khandwa-2 (0.34), RAJ-2 (5.10), L-761 (5.43), GJHV-358 (6.11) rate of reduction was less than moisture stress susceptible MCU-5 (16.47). Genotypes with higher chlorophyll recorded higher yield under moisture stress, Cetin et al., (2009) 
revealed that leaf chlorophyll content may be one of the major factors influencing seed cotton yield of cotton under drought stress.

As normal phenomenon, proline is acting as an osmoprotectant, in the moisture stress condition (Bates et al., 1973). Singh et al., (1992) reported that under water stress condition, reduction RWC and increased leaf proline content was observed in drought tolerant cotton variety (SRT 1). Similarly, in this study also, under moisture stress situation reduced RWC rate was observed at 80-82 DAS with higher proline rate (Table 7) in genotypes, GJHV-358 (119.25), PH1009 (76.37), CCH1831 (97.02), Khandwa-2 (119.7) and RAJ-2 (93.37) than susceptible MCU-5 (38.55). The increased proline content under moisture stress condition was found in many studies (Parida et al., 2007; Aktas et al., 2009; Amudha et al., 2014). This is due to the fact that drought tolerant plants can regulate their solute potential to compensate for transient or extended periods of water stress by the process called osmotic adjustment and proline acts as an osmoregulator and cellular protectant under moisture stress (Hanower and Brzozowska, 1975; Patil et al., 2011). Moisture stress induces oxidative stress leads to increased production of reactive oxygen species (ROS) like superoxide $\left(\mathrm{O}_{2}{ }^{-}\right)$, hydroxyl radical $\left(\mathrm{OH}^{-}\right)$ and hydrogen peroxide $\left(\mathrm{H}_{2} \mathrm{O}_{2}\right)$, which can attack lipids, proteins, carbohydrates, and nucleic acids of plant system (Smirnoff, 1993). However there were several enzymes that detoxify ROS resulting from stress. Superoxide dismutase (SOD) is the first defense enzyme that converts superoxide to $\mathrm{H}_{2} \mathrm{O}_{2}$, which can be scavenged by catalase (CAT) (Hosseini et al., 2015). Hydrogen peroxide is produced in the process of stress and is detoxified in living organisms by catalase and glutathione peroxidase (Jin et al., 2006). In the present study, increase in peroxidase activity in cotton genotypes (Table
7) under moisture stress was recorded than normal condition and the rate of increase in peroxidase enzyme in moisture stress tolerant genotype RAJ-2 (118.03), Bikaneri nerma (91.58), 5433A2A03N83 (85.42) was greater than susceptible MCU-5 (3.01).

\section{Yield and yield contributing traits}

The ANOVA showed significant variation in irrespective of genotypes between conditions (Table 8) for yield contributed traits. When data was analyzed in individually condition wise, under moisture stress condition significant difference among genotypes was observed for all studied productivity traits except sympodia (Table 9). In many studies, yield and yield contributed traits have been found to reduce under moisture stress condition (Luz et al., 1997; Osborne and Banks, 2006; Karademir et al., 2011; Sahito et al., 2015).

However the rate of reduction in moisture stress condition than control condition is vary among genotypes and Karademir et al., (2011) reported, that in drought tolerant varieties less yield reduction observed than susceptible varieties. In the present study (Table 10). The genotypes, L-761 (13.31), GJHV-358 (14.08), RS-810 (14.32), Khandwa-2 (18.31), CCH1831 (20.01) recorded lower reduction of yield than susceptible MCU-5 (52.32). Yield is a complex trait and is the end product of various developmental and physiological processes. Therefore in the present study, as compared to control condition, under moisture stress condition, cotton yield was reduced due to changes in physio-biochemical process. At 80-82 DAS (30 days water withholding), drought tolerant genotypes recorded higher relative water content, stomatal conductance, photosynthetic rate and transpiration rate with increasing canopy temperature than susceptible MCU-5, resulted 
in higher yield in moisture stress tolerant genotypes (Khandwa-2, RAJ-2, CCH1831, PH1009, and RHC0811) than susceptible MCU-5. Ephrath (1990); Genty (1987); Marani (1985); Turner et al., (1986) reviewed that adequate water availability in plant system is the main factor maintained photosynthesis and stomatal conductance and, consequently positively correlated with increased yields in drought tolerant genotypes than susceptible (Fischer et al., 1998). Although several physiological traits studied in relation to yield potential, but no single parameter has been identified as the sole trait of improving yield (Barbour et al., 2000; Bray, 1993; Radin et al., 1994; Ullah et al., 2008) in moisture stress induction.

Therefore, in this study, the maximum yield of desirable drought tolerant genotypes showed variation for possible traits like sympodial number, number of fruiting points, number of bolls and plant height and these productivity traits were ultimately affected by physio-biochemical plant response. Higher chlorophyll content and photosynthetic rate recorded in Khandwa-2 and higher stomatal conductance, RWC recorded in genotypes F2226 and Bikaneri nerma than susceptible MCU-5 in same level of moisture stress condition and it leads to maximum number of bolls in moisture stress condition than susceptible MCU-5. It indicates that significant difference between genotypes for moisture stress tolerance is regulated by different metabolic processes under stress condition.

The tolerance of genotypes to moisture stress also result in increasing antioxidants, proline and peroxidase enzyme. All their activities were higher in drought tolerant genotypes as compared to susceptible, MCU-5. Khatun et al., (2008) reported that antioxidant peroxidase minimize cellular damage which caused by reactive oxygen species (ROS) such as superoxide, perhydroxy radicals, hydrogen peroxide, hydroxy radicals and results in to higher yield in tolerant genotypes grown in water deficit condition. Meanwhile, Ullah et al., (2017); Zhang et al., (2014) revealed that antioxidant enzyme activities, decreased in drought susceptible genotypes and increased in drought tolerant genotypes which is responsible for higher yield potential in stress condition.

When water-deficit stress occurs during the square formation stage, reduction in seed cotton yield of cotton genotypes is mainly due to square and young boll shedding (Cook and El-Zik, 1992). Other study reports Alishah and Ahmadikhah (2009); Khorgade and Ekbote (1980); Karademi et al., (2011); Zare et al., (2014) evaluated cotton genotypes in moisture stress condition and recorded reported higher seed cotton yield in stress condition due to maintenance of higher water use efficiency and other physiological parameter. The higher productivity traits were related to plant physio-biochemical metabolic process. Therefore in this study, under moisture stress condition significant variation between genotypes for physio-biochemical traits associated with variation in number of fruiting bodies, boll number, plant height and sympodial number was observed.

Substantial genotypic variation found for physio-biochemical attributes among the cotton genotypes and as expected higher rate of RWC, lower transpiration rate, higher photosynthetic rate, proline content and peroxidase enzyme activity would record higher yield in genotypes Khandwa-2, F2226, 5433A2A03N83, RAJ-2, and RHC0811 under moisture stress condition. The moisture stress tolerance due to regulation of physiobiochemical metabolic process results in to less reduction of yield in genotypes, GJHV358 (14.08), RS-810 (14.32), Khandwa-2 (18.31) and $\mathrm{CCH} 1831$ (20.01) than susceptible MCU-5. 


\section{References}

Ahuja, S.L. and O.P. Tuteja, 2001, Variability studies for yield, its components and physiological attributes under stress condition in Gossypium hirsutum cotton. J. Cotton Res. and Dev., 15 (1): 15-18.

AICCIP Annual report, 2016, [All India Coordinated Cotton Improvement Project].

Aktas, L. Y., S. Dagnon, A. Gurel, E. Gesheva, and A. Edreva, 2009, Drought tolerance in cotton: involvement of non-enzymatic ROS-scavenging compounds J. Agro. Crop Sci., 195: 247-253.

Alishaha, A. O. and A. Ahmadikhahb, 2009, The effects of drought stress on improved cotton varieties in golesatn province of Iran. Int. J. Pl. Prod., 3 (1): 17-26.

Amudha. J., S. Chhajed, and G. Balasubramani, 2014, Cotton Transgenic Plants with DreBinding Transcription Factor Gene (DREB1A) Confers Enhanced Tolerance to Drought. Int. J. Adv. Biotechnol. Res. 15(4): 635-648.

Ananthi, K. and H. Vijayaraghavan, 2012, Development of drought tolerant index in cotton genotypes based on relative water content and yield. Asian J. Bio. Sci., 7(2): 138-144.

Barbour, M. M., R.A. Fischer, K.D. Sayer, and G.D. Farquhar, 2000, Oxygen isotope ratio of leaf and grain material correlates with stomatal conductance and grain yield in irrigated wheat. Australian J. Pl. Physiol., 27: 625-637.

Barrs, H. D. and P.E. Weatherley, 1962, A reexamination of the relative turgidity technique for estimating water deficits in leaves. Australian J. Biol. Sci., 15: 413-428.

Bates, L. S., 1973, Rapid determination of free proline for water-stress studies. Plant Soil, 39: 205-207.

Belko, N., M. Zaman-Allah, N.N. Diop, N. Cisse, G. Zombre, J.D. Ehlers, and V. Vadez, 2012, Restriction of transpiration rate under high vapour pressure deficit and nonlimiting water conditions is important for terminal drought tolerance in cowpea. $\mathrm{Pl}$. Biol., 15: 304-316.
Berger, B., B. Parent, and M. Tester, 2010, High throughput shoot imaging to study drought responses. J. Exp. Bot., 61: 3519-3528.

Blum, A., 1988, Plant breeding for stress environments. CRC Press, Florida.

Borgo, L., C.J. Marur, and L.G.E. Vieira, 2015, Effects of high proline accumulation on chloroplast and mitochondrial ultrastructure and on osmotic adjustment in tobacco plants. Acta Science, 37: 191-199.

Bray, E. A., J. Bailey-Serres, and E. Weretilnyk. 2000, Response to abiotic stresses. p. 11581203. In B.B. Buchanan, W. Gruissem, and R.L. Jones (eds.) Biochemistry and molecular biology of plants. American Society of Plant Physiologists, Rockville, MD.

Brito, G., V. Sofiatti, M.M.A. Lima, L.P. Carvalho, and J.L.S. Filho, 2011, Physiological traits for drought phenotyping in cotton. Acta Scientiarum. Agron., 33(1): 117-125.

Cetin, K., K. Emine, E. Remzi, and G. Oktay, 2009, Correlations and Path Coefficient Analysis between Leaf Chlorophyll Content, Yield and Yield Components in Cotton (G. hirsutum L.) under Drought Stress Conditions. Notulae Botanicae Horti Agrobotanici Cluj-Napoca, 37: 241-244.

Chaves, M. M. 1991. Effects of water deficits on carbon assimilation. J. Exp. Bot., 42: 1- 16.

Conaty, W. C., J.R. Mahan, J.E. Neilsen, D.K.Y. Tan, S.J. Yeates, and B.G. Sutton, 2015, The relationship between cotton canopy temperature and yield, fibre quality and water use efficiency. Field Crops Res., 183: 329-342.

Constable, G.A., and A.B. Hearn, 1981, Irrigation of crops in a sub-humid climate. VI. Effects of irrigation and nitrogen fertilizer on growth, yield and quality of cotton. Irrig. Science, 3:17-28.

Cook, C.G., and K.M. el Zik, 1992, Cotton seedling and first-bloom plant characteristics: relationships with droughtinfluenced boll abscission and lint yield. Crop Sci., 32: 1464-1467.

Cornic, G., and A. Massacci, 1996, Leaf photosynthesis under drought stress. Neil R. Baker (ed): Photosynthesis and the Environment, pp. 347-366. 
Costa, V.A., and J.T. Cothren, 2011, Drought effects on gas exchange, chlorophyll, and plant growth of 1-methylcyclopropene treated cotton. Agron. J., 103: 1230-1241.

Cull, P.O., A. B. Hearn, and R.C.G. Smith, 1981, Irrigation scheduling of cotton in a climate with uncertain rainfall. I. Crop water requirements and response to irrigation. Irrig. Science, 2:127-140.

Deeba, F., A.K. Pandey, S. Ranjan, A. Mishra, R. Singh, Y.K. Sharma, P.A. Shirke, and V. Pandey. 2012. Physiological and proteomic responses of cotton (Gossypium herbaceum L.) to drought stress. Plant Physiol. Biochem., 53: 6-18.

Ennahli, S., and H.J. Earl, 2005, Physiological limitations to photosynthetic carbon assimilation in cotton under water stress. Crop Science, 45: 2374-2382.

Ephrath, J., A. Marani, and B.A. Bravdo, 1990, Effect of moisture stress on stomatal resistance and photosynthetic rate in cotton (Gossypium hirsutum L.): Controlled level of stress. Field Crops Res., 23: 117-131.

Farquhar, G.D., and R.A. Richards, 1984, Isotopic composition of plant carbon correlates with water use efficiency of wheat genotypes. Astralian J. Pl. Physiol., 11: 39-552.

Fischer, R.A., and R. Maurer, 1978. Drought resistance in spring wheat cultivars: I. Grain yield responses. Aus. J. Agricul. Res., 29: 897-907.

Fumis, T.F., and J.F. Pedras. 2002. Variação nos níveis de prolina, diamina e poliaminas em cultivares de trigo submetidas a déficits hídricos. (In Portuguese, with English abstract.) Pesq. Agropec. Bras., 37: 449459.

Genty, B., J.M. Briantais, and J.B.V. Dasilva, 1987, Effects of drought on primary photosynthetic processes of cotton leaves. Plant Physiol., 83: 360-364.

Gerik, T.J., K.L. Faver, P.M. Thaxton, and K.M. El-zik, 1996, Late season water stress in cotton I. Plant growth, water use and yield. Crop Sci., 36(4): 914-921.

Gorham, J., J. Bridher, M.N. Malik, and I.A. Khan, 1998, Physiological responses of cotton to water deficits in Pakistan. In: Proc. of World Cotton Res Conference-2,
September, 6-12, Athens, Greece, pp. 557590.

Grimes, D.W., W.L. Dickens, and H. Yamada, 1978, Early season water management for cotton. Agron. J., 70: 1009-1012.

Hanower, P., and J. Brzozowska, 1975, Effects of osmotic stress on composition of free amino acids in cotton leaves. Phytochem., 14: 1691-1694.

Hassan, H. M., F.M. Azhar, A.A. Khan, S.M.A. Basra, and M. Hussain, 2015, Characterization of cotton (Gossypium hirsutum) germplasm for drought tolerance using seedling traits and molecular markers. Int. J. Agric. Biol., 17: 1213-1218.

Hatfield, J.L., J.E. Quinsenberry, and R.E. Dilbeck, 1987, Use of canopy temperature to identify water conservation in cotton germplasm. Crop Sci., 27: 269-273.

Hosseini, S.M., T. Hasanloo, and S. Mohammadi, 2015, Physiological characteristics, antioxidant enzyme activities, and gene expression in 2 spring canola (Brassica napus L.) cultivars under drought stress conditions Turkish J. Agri. For., 39: 413420.

Imran, M., A. Shakee, F.M. Azhar, M.F. Saleem, A. Saeed, W. Nazeer, M. Riaz, M. Naeem, and A. Javaid, 2012, Combining ability analysis for within-boll yield components in upland cotton (G. hirsutum L.). Genetic and Molecul. Res., 11(3): 2790-2800.

Isoda, A., and P. Wang, 2002, Leaf temperature and transpiration of field grown cotton and soybean under arid and humid conditions. Plant Prod. Sci. 5(3): 224-228.

Jamal, A., M.N. Shahid, B. Aftab, B. Rashid, S. Kiani, B.B. Mohammed, M.B. Sarwar, S. Hassan, and T. Husnain, 2015, Alterations in photosynthetic, water relations and biochemical components in cotton subjected to drought stress. J. Global Biosci., 4: 1517- 1529.

Jenks M.A., L. Andersen, R.S. Teusink, M.H. Williams, 2001, Leaf cuticular waxes of potted rose cultivars as affected by plant development, drought and paclobutrazol treatments. Physiol. Plant, 112: 62-70.

Jin, J., N. Shan, N. Ma, J. Bai, and J. Gao, 2006, Regulation of ascorbate peroxidase at the transcript level is involved in tolerance to 
postharvest drought stress in the cut rose (Rosa hybrida L.) cv. Samantha. Post. harvest Biol. Tec., 40: 236-243.

Kaiser, W.M. 1987, Effect of water deficit on photosynthetic capacity. Physiologia Plantarum, 71: 142-149.

Kamaran, S., Imran, M., Khan, T.M., Munir, M.Z., Rashid, M.A., Muneer, M.A., 2016, Genetic studies of genotypic responses to water stress in upland cotton (Gossypium hirsutum L.). Int. J. Agron. Agril. Res., 8(6): 1-9.

Karademir, C., E. Karademir, R. Ekinci, and K. Berekatoglu, 2011, Yield and fiber quality properties of cotton ( $G$. hirsutum L.) under water stress and non-stress conditions. African J. Biotechnol., 10(59): 1257512583.

Karademir, C., E. Karademir, R. Ekinci, and O. Gencer, 2009, Correlations and path coefficient analysis between leaf chlorophyll content, yield and yield components in cotton ( $G$. hirsutum L.) under drought stress conditions. Not. Bot. Hort. Agrobot. Cluj., 37(2): 241-244.

Khan, C.S.Z., W. Spreer, Y. Pengnian, X. Zhao, H. Othmanli, X. He, and J. Muller, 2015, Effect of dust deposition on stomatal conductance and leaf temperature of cotton in northwest. Water, 7: 116-131.

Khatun, S., M.B. Ali, E.J. Hahn, and Y. Paek, 2008, Copper toxicity in Withania somnifera: growth and antioxidant enzymes responses of in vitro grown plants. Environ Exp. Bot., 64: 278-295.

Khorgade, P.W. and A.P. Ekbote, 1980, Path coefficient analysis in upland cotton. Ind. J. Agri. Sci., 50: 6-8.

Kosma, D.K., and M.A. Jenks, 2007, Ecophysiological and molecular-genetic determinants of plant cuticle function in drought and salt stress tolerance. In MA Jenks, PM Hasegawa, SM Jain, eds, Advances in Molecular Breeding toward Drought and Salt Tolerant Crops. Springer, Dordrecht, The Netherlands, pp 91-120

Kramer, P.J. 1983. Water relation of plants. Academic Press. Inc London

Laffray, D. and P. Louguet, 1990, Stomatal response and drought resistance. Bulletin de la Societe Botanique de France-actualites Botaniques, 137: 47-60.

Lahong, Y., Z. Qiang, H.T. Mao, L.Z. Yq, and T.M. Hua, 2000, The effect of soil moisture on the chlorophyll content and photosynthetic rate of different cotton varieties. China Cottons, 27(2): 21-22.

Leidi, E.O., M. Lopez, and J.C. Gutierrez, 1993. Searching for tolerance to water stress in cotton genotypes: Photosynthesis, stomatal conductance and transpiration. Photosynthetica, 28: 383-390.

Li, D., C. Li, H. Sun, L. Liu, and Y. Zhang, 2012, Photosynthetic and chlorophyll fluorescence regulation of upland cotton (Gossiypium hirsutum L.) under drought conditions. Plant Omics J., 5: 432-437.

Li, P. C., H.L. Dong, A.Z. Liu, J.R. Liu, M. Sun, G.P. Wang, S.P. Zhang, Y.B. Li, and S.C. Mao, 2014, Diagnosis of premature senescence of cotton using SPAD value. Agril. Sci., 5: 992-999.

Loka, D. A., 2012, Effect of water-deficit stress on cotton during reproductive development. Ph.D. Dissertation, University of Arkansas, Fayetteville, Ark.

Lopez, I.M., J.C. Gutierrez, and E.O. Leidi, 1995, Selection of characterization of cotton cultivars for dry land production in the southwest of Spain. European J. Agron., 4(1): 119-126.

Lugojan, C. and S. Ciulca 2011, Evaluation of relative water content in winter wheat, $J$. Hort. For. Biotech., 15(2): 173- 177.

Luz, M., J.R.C. Bererra, A.N. Barreto, J.W.D. Santosh, N.M. Amorim, and M.J. Luz, 1997, Effect of water stress on cotton yield and fibre quality. Revista de Oleaginosas Fibrosas., 1 (1): 125-133.

Malik, T.A., D. Wright, and D.H. Virk, 1999, Inheritance of net photosynthesis and transpiration efficiency in spring wheat, Triticum aestivum L., under drought. Plant Breed., 118: 93-95.

Marani, A., D.N. Baker, V.R. Reddy and Mckinion, 1985, Effect of water stress on canopy senescence and carbon exchange rates in cotton. Crop Sci., 25(5): 798-802.

Marechaux, I., M.K. Bartlett, L. Sack, C. Baraloto, J. Engel, E. Joetzjer, and J. Chave. 2015. Drought tolerance as 
predicted by leaf water potential at turgor loss point varies strongly across species within an Amazonian forest. Funct. Ecol., pp1-10.

Massacci, A., S.M. Nabiev, L. Pietrosanti, S.K. Nematov, T.N. Chernikova, K. Thor, and J. Leipner, 2008, Response of the photosynthetic apparatus of cotton $(G$. hirsutum) to the onset of drought stress under field conditions studied by gasexchange analysis and chlorophyll fluorescence imaging. Plant Physiol. Biochem., 46: 189-195.

Meyer, W.S. and S. Walker, 1981, Leaflet orientation in water stressed soybeans. Agron. J., 73: 1071-1074.

Michael, B.L., W.R. Jordan, and R.D. Powell, 1973, Abscission processes in cotton: Induction by plant water deficit. Agron. J., 65: 202-204.

Nepomuceno, A.L., D.M. Oosterhuis, and J.M. Stewart, 1998, Physiological responses of cotton leaves and roots to water deficit induced by polyethylene glycol. Environ. Exper. Bot., 40: 29-41.

Osborne, S. and J.C. Banks, 2006, The effect of water stress during bloom on lint yield, fibre quality and price, Beltwide Cotton Conferences, San Antonio, Taxas, January, 3-6, pp. 1679-1780.

Panse, V.G. and P.V. Sukhatme, 1967, Statistical methods for agricultural workers (pp. 167174). New Delhi: ICAR Publication

Parida, A.K., S.D. Vipin, S.P. Manoj, G.V. Umalkar, and P.A. Laxman, 2007, Alterations in photosynthetic pigments, protein and osmotic components in cotton genotypes subjected to short-term drought stress followed by recovery. Plant Biotechnol. Rep., 1: 37-48.

Passioura, J.B., 1982, Water in the Soil-PlantAtmosphere Continuum. O. L. Lange et al., (eds.), Physiological Plant Ecology II (C) Springer-Verlag Berlin Heidelberg.

Patil, M. S. 2011, Refining info-crop model for drought varieties in cotton. M. Sc. (Agri.) Thesis, Univ. Agric. Sci., Dharwad, Karnataka (India).

Pettigrew, W. T. 2004, Physiological consequences of moisture deficit stress in cotton. Crop Sci., 44: 1265-1272.
Quisenberry, J.E., C.E. Wendt, J.D. Berun, and B.L. Mcmichel, 1985, Potential for using leaf turgidity to select drought tolerance in cotton. Crop Sci., 25: 294-298.

Radin, J.W., A.W. Hartung, B.A. Kimball, and J.R. Mauney, 1988, Correlation of stomatal conductance with photosynthetic capacity of cotton only in a co2-enriched atmosphere: mediation by abscisic acid? Plant Physiol., 88: 1058-1062.

Rahman, M., I. Ullaha, M. Ahsraf, J.M. Stewart, and Y. Zafara, 2008, Genotypic variation for drought tolerance in cotton, Agron. Sustain. Dev., 28: 439-447

Rahman, S., M.S. Shaheen, M. Rahman, and T.A. Malik, 2000, Evaluation of excised leaf water loss and relative water content as screening techniques for breeding drought resistant wheat. Pak. J. Biol. Sci., 3: 663665

Rebetzke, G.J., A.R. Rattey, G.D. Farquhar, R.A. Richards, and A.G. Condon, 2013, Genomic regions for canopy temperature and their genetic association with stomatal conductance and grain yield in wheat. Funct. Plant Biol., 40: 14-33.

Reddy, A.R., K.V. Chaitanya, and M. Vivekanandan 2004, Drought-induced responses of photosynthesis and antioxidant metabolism in higher plants. J. Plant Physiol., 161: 1189-1202.

Reddy, K.R. and H.F. Hodges 1996, Mepiquat chloride (PIX)-induced changes in photosynthesis and growth of cotton A. Ramachandra Plant Growth Reg., 20: 179183.

Roberts, D.R. and E.B. Dumbroff, 1986, Relationships among drought resistance, transpiration rates and abscisic acid levels in three northern conifers. Tree Physiol., 1: 161-167.

Rosielle, A.A. and J. Hamblin, 1981. Theoretical aspects for yield in stress and non-stress environments. Crop Science, 21: 943-946.

Sahito, A., Z.A. Baloch, A. Mahar, S.A. Otho, S.A. Kalhoro, A. Ali, F.A. Kalhoro, R.N. Soomro, and F. Ali, 2015, Effect of water stress on the growth and yield of cotton crop (Gossypium hirsutum L.). American J. Plant Sci., 6: 1027-1039. 
Santos, T.B., I.G. Budzinski, C.J. Marur, C.L. Petkowicz, L.F. Pereira, and L.G. Vieira, 2011, Expression of three galactinol synthase isoforms in Coffea arabica L. and accumulation of raffinose and stachyose in response to abiotic stresses. Plant Physiol. Biochem., 49: 441-448.

Schroeder J.I., J.M. Kwak, and G.J. Allen, 2001, Guard cell abscisic acid signalling and engineering drought hardiness in plants. Nature., 410(6826): 327-30.

Singh, D., R.K. Sahay, and M. Singh, 1992, Drought stress in cotton: Identification of critical growth stage. Pl. Physiol. Bochem., 19(1): $55-57$

Singh, S.P., 1975, Studies on the effect of soil moisture stress on the yield of cotton. Ind. J. Pl. Physiol., 18: 49-55.

Tezara, W., V.J. Mitchell, and S.D. Driscoll, 1999, Water stress inhibits plant photosynthesis by decreasing coupling factor and ATP. Nature. 401: 914-917.

Turner, N.C., A.B. Hearn, J.E. Begg, and G.A. Constable 1986. Cotton (G. hirsutum L.) physiological and morphological responses to water deficits and their relationship to yield. Field Crops Res., 14: 153-170.

Ullah, A., H. Sun, X. Yang, and X. Zha, 2017, Drought coping strategies in cotton: increased crop per drop. Plant Biotechnol. J., 15: 271-284.

Ullah, I., M. Rahmana, M. Ashraf, and Y. Zafar, 2008, Genotypic variation for drought tolerance in cotton (Gossypium hirsutum L.): Leaf gas exchange and productivity. Flora, 203: 105-115.

Wang, X., H. Xie, H. Guan, and X. Zhou, 2007, Different responses of MODIS-derived
NDVI to root-zone soil moisture in semiarid and humid regions. J. Hydrology, 340: $12-24$.

Williams M.H., E., Rosenqvist, and M. Buchhave, 1999, Response of potted miniature roses (Rosa $\mathrm{x}$ hybrida) to reduced water availability during production. J Hortic Sci Biotechno., l 74: 301-308.

Yi, L., Y. Zhou, and T. Hua, 2000, The effect of soil moisture on the chlorophyll content and photosynthetic rate of different cotton varieties. China Cottons, 27: 21-22.

Zaman-Allah, M., D.M. Jenkinson, and V. Vadez, 2011, A conservative pattern of water use, rather than deep or profuse rooting, is critical for the terminal drought tolerance of chickpea. Funct. Plant Biol., 38: 270-281.

Zare, M., G.R. Mohammadifard, F. Bazrafshan, and M. Zadehbagheri, 2014, Evaluation of cotton (Gossypium hirsutum L.) genotypes to drought stress. Int. J. Biosci., 4 (12): 158166.

Zhang, L., J. Peng, T. T. Chen, X. H. Zhao, S. P. Zhang, S. D. Liu, H. L. Dong, L. Feng, and S. X. Yu, 2014, Effect of drought stress on lipid peroxidation and proline content in cotton roots. J. Animal Plant Sciences, 24(6): 1729-1736.

Zhang, Y.L., H.Z. Zhang, M.W. Du, W. Li, H.H. Luo, W.S. Chow, and W.F. Zhan, 2010, Leaf wilting movement can protect waterstressed cotton (Gossypium hirsutum L.) plants against photo inhibition of photosynthesis and maintain carbon assimilation in the field. J. Plant Bio., 53(1): 52-60.

\section{How to cite this article:}

Thakur Pranita Prabhakar, D.P. Biradar and Katageri, I.S. 2018. Effect of Physio-biochemical Factors Influencing Moisture Stress Tolerance in Cotton (Gossypium hirsutum L.). Int.J.Curr.Microbiol.App.Sci. 7(03): 619-637. doi: https://doi.org/10.20546/ijcmas.2018.703.074 